\title{
THERMODYNAMICS OF SURFACE PHENOMENA
}

\author{
J. C. Melrose \\ Mobil Research and Development Corporation, \\ P.O. Box 900, Dallas, Texas 75221, U.S.A.
}

\begin{abstract}
The thermodynamic treatment of the interfacial region corresponding to two fluid phases in contact is discussed. The features of this analysis which are reviewed include the classical treatment of Gibbs and the extensions to this treatment which are due to Buff. It is shown that these extensions are essential if the logical structure of the analysis is to be regarded as complete.
\end{abstract}

\section{INTRODUCTION}

The thin, nonhomogeneous region separating two homogeneous bulk phases in contact constitutes an interface. It is generally recognized that an adequate thermodynamic treatment of such a region must be based on the work of Gibbs ${ }^{1}$. Nevertheless, the literature contains a number of proposals for modifying various features of this treatment. It is, in fact, remarkable that no other important contribution of Gibbs to the understanding of the equilibrium states of heterogeneous substances has given rise to so many reservations and attempts to develop alternative treatments.

The proposed modifications are usually concerned with one or more of several concepts which are characteristic of the Gibbsian treatment. The first of these concepts involves the notion of a mathematical dividing or reference surface, located within or very near the nonhomogeneous interfacial region. This surface serves to define the geometrical configuration of the interfacial region and also partitions the volume of the system between the two bulk phases. A second feature of the Gibbs treatment which is occasionally challenged is the definition of the chemical potentials which are appropriate to the components present in an interfacial region. Finally, the nature of the interdependence between the parameter referred to as the-interfacial or surface tension and the geometrical configuration itself has been the source of much conflicting opinion.

It is one of the purposes of this review to discuss in detail the principal area of conceptual difficulty in the Gibbsian treatment. It is concluded that in this instance the classical approach of Gibbs, when properly interpreted, plays an essential role in providing generality as well as precision to the resulting formalism. A further purpose will be to discuss the recent contributions of Buff $^{2}$ to the general phenomenological theory of fluid-fluid interfacial regions. On the basis of these contributions, which involve concepts not traditionally regarded as thermodynamic in nature, it is 


\section{J. C. MELROSE}

possible to provide a substantial extension of the Gibbsian treatment. In fact, it is seen that the various difficulties which have given rise to proposed modifications are in part due to the incomplete nature of the Gibbsian approach.

A detailed exposition of the Gibbsian or strictly thermodynamic treatment of interfacial regions is to be found in the treatise by Defay et al. ${ }^{3}$. The review articles by Buff ${ }^{4}$ and by Ono and Kondo include, in addition to the thermodynamic analysis, accounts of the manner in which the condition for hydrostatic equilibrium provides an essential feature of the phenomenological theory. The treatment of Ono and Kondo, like that of Tolman ${ }^{6}$, Koenig ${ }^{7}$ and $\mathrm{Hill}^{8}$, is restricted to interfaces of spherical shape, whereas Buff's treatment ${ }^{2,4}$ applies to surfaces of nonspherical configuration. The following discussion will rely extensively on a recent review ${ }^{9}$ of Buff's work.

\section{THE DIVIDING SURFACE AND GIBBS EXCESS QUANTITIES}

The thermodynamic analysis which will be developed is intended to describe a system such as is represented in Figure 1. Two homogeneous fluid phases $\alpha$ and $\beta$ are taken to be in contact, and external macroscopic fields are

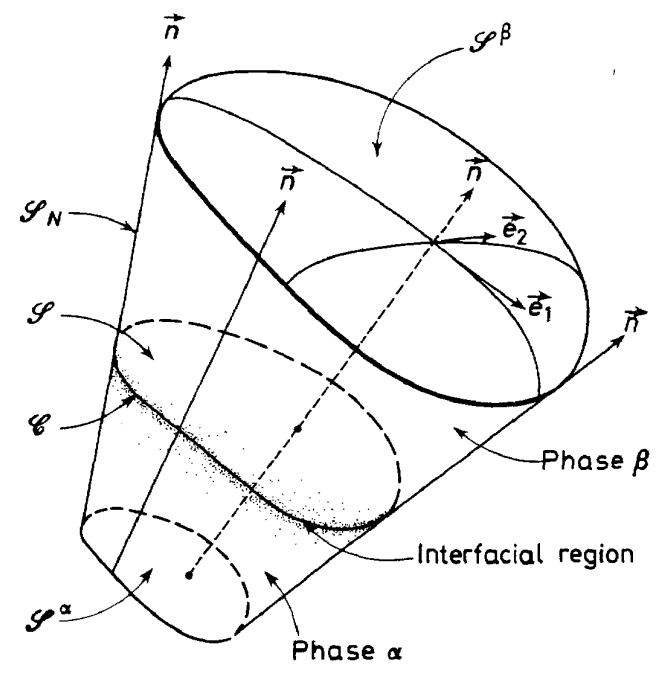

Figure 1. System with curved fluid-fluid interfacial region

assumed to be absent. Within the thin, nonhomogeneous region which separates $\alpha$ and $\beta$ a mathematical surface, denoted as $\mathscr{S}$, is placed. This surface will in general be curved, since its geometrical shape or configuration is determined by the configuration of the interfacial region. The nature of the physical conditions which determine the latter will emerge at a further stage of the analysis.

Whatever the configuration of the interfacial region may be, the configuration of $\mathscr{S}$ is taken to be such that the variation of the point density of matter 
along a normal to $\mathscr{S}$ is sensibly invariant over the entire set of normal directions. This is possible only if the interfacial region is assumed to be homogeneous in a two-dimensional sense. The position of $\mathscr{S}$ with respect to the variation in the density in any given normal direction through the interfacial region is arbitrary. Consequently, various conventions may be used in order to fix its position with respect to an origin selected on a given normal. It is assumed, following Buff ${ }^{2,4}$, that the various positions so chosen then establish a set of surfaces which are parallel in the mathematical sense ${ }^{10,11}$.

Without loss of generality, the external boundaries of the system may be considered to be determined, first, by specifying a closed curve $\mathscr{C}$ lying within $\mathscr{S}$. The set of normals to $\mathscr{P}$ passing through the curve $\mathscr{C}$ then forms a surface $\mathscr{S}_{N}$. Secondly, two surfaces, $\mathscr{S}^{\alpha}$ and $\mathscr{S}^{\beta}$, parallel to and lying on either side of $\mathscr{S}$, are specified to be sufficiently far from $\mathscr{S}$ that in their vicinity the point densities of matter are uniform. Thus, $\mathscr{S}^{\alpha}$ and $\mathscr{S}^{\beta}$ lie entirely within the homogeneous phases $\alpha$ and $\beta$, respectively. The matter enclosed within the three surfaces, $\mathscr{S}^{\alpha}, \mathscr{S}^{\beta}$ and $\mathscr{S}_{N}$, then constitutes the system $\mathscr{B}$. This system is considered to be an open system in the usual thermodynamic sense.

A basic assumption underlying the Gibbsian approach just outlined is that the densities of the extensive thermodynamic quantities vary in a continuous manner along a coordinate which is normal to the interfacial region. The parameters in question are the internal energy, $U$, the entropy, $S$, and the number of moles of each component, $N_{i}(i=1 \ldots \kappa)$. If $M$ is taken as denoting each of these extensive quantities and $\dot{M}$ as the density, i.e. the limit of $(M / V)$ as the volume, $V$, vanishes, the corresponding surface excess quantity can be written as

$$
m=\int_{\lambda^{\alpha}}^{\lambda^{\beta}}\left\{\hat{M}(\lambda)-\hat{M}^{\alpha \beta}\left(\lambda^{0}\right)\right\} B\left(\lambda, \lambda^{0}\right) \mathrm{d} \lambda
$$

Here $\lambda$ measures the distance along the normal coordinate from some arbitrary origin, and $\lambda^{0}$ then specifies the position of the Gibbs dividing or reference surface $\mathscr{S}$ (see Figure 2). The area of the reference surface $\mathscr{S}$ is denoted by $\Omega$. The quantity $\hat{M}^{\alpha \beta}\left(\lambda^{0}\right)$ is given by

$$
\hat{M}^{\alpha \beta}\left(\lambda^{0}\right)=\{1-A(\lambda)\} \hat{M}^{\alpha}+A(\lambda) \hat{M}^{\beta}
$$

where $A(\lambda)$ is a unit step function,

$$
A(\lambda)=0 \text { for } \lambda<\lambda^{0} ; A(\lambda)=1 \text { for } \lambda \geqslant \lambda^{0}
$$

and $\hat{M}^{\alpha}$ and $\hat{M}^{\beta}$ are the densities characteristic of the regions of the bulk phases in which homogeneity can be assumed. Clearly, the limits of the integration in equation 2.1 need only be extended to such regions, i.e. to the surfaces $\mathscr{S}^{\alpha}$ and $\mathscr{S}^{\beta}$.

The quantity $B\left(\lambda, \lambda^{0}\right)$ in equation 2.1 is a factor accounting for the change in area of the surface on which the function $\hat{M}(\lambda)$ is defined and is given by

$$
B\left(\lambda, \lambda^{0}\right)=1+\left(\lambda-\lambda^{0}\right) J+\left(\lambda-\lambda^{0}\right)^{2} K
$$

Here, $J$ and $K$ are the mean and Gaussian curvatures ${ }^{10,11}$ characterizing an infinitesimal area, $\delta \Omega$, on the reference surface $\mathscr{S}$. A restriction on the variation of the mean curvature $J$ from point to point on such a surface will be intro- 


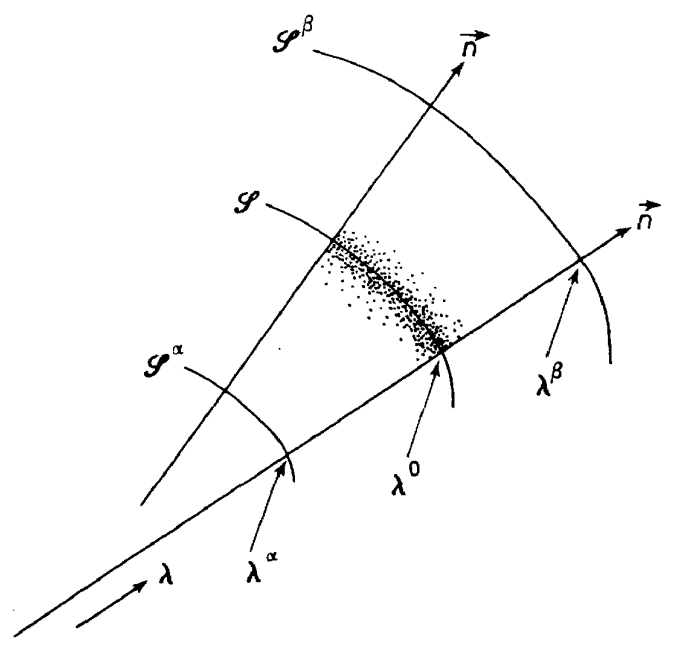

Figure 2. Normal coordinate for parallel surfaces

duced later. This restriction and the small magnitude, as compared with $J^{-1}$ and $K^{-\frac{1}{2}}$, of the distance over which $\hat{M}$ and $\hat{M}^{\alpha \beta}$ differ appreciably are sufficient to ensure that the surface density, $m$, will be very nearly uniform over the entire interfacial region. Thus, the two-dimensional homogeneity assumed for the density profile $\hat{M}(\lambda)$, where $M=N$, the total number of moles, holds to a high degree of approximation for each of the densities, $m$.

In order to specify the volumes $V^{\alpha}$ and $V^{\beta}$ into which the reference surface $\mathscr{S}$ partitions the total volume of the system, integrals similar to that of equation 2.1 are required:

$$
\begin{aligned}
& V^{\alpha}=\int_{0}^{\Omega} \int_{\lambda^{\alpha}}^{\lambda^{0}} B\left(\lambda, \lambda^{0}\right) \mathrm{d} \lambda \mathrm{d} \Omega \\
& V^{\beta}=\int_{0}^{\Omega} \int_{\lambda^{0}}^{\lambda^{\beta}} B\left(\lambda, \lambda^{0}\right) \mathrm{d} \lambda \mathrm{d} \Omega
\end{aligned}
$$

If now two sets of extensive parameters denoted as $M^{\alpha}$ and $M^{\beta}$ are defined by the following:

$$
M^{\alpha}=V^{\alpha} \hat{M}^{\alpha} ; \quad M^{\beta}=V^{\beta} \hat{M}^{\beta}
$$

it is clear that, if the area $\Omega$ is sufficiently small that $J$ and $K$ are constant, the total quantity $M$ must be given by

$$
M=M^{\alpha}+M^{\beta}+m \Omega
$$

Corresponding to the various extensive quantities denoted by $M$, equation 2.1 provides a definition of the surface densities of interfacial energy, $u$, entropy, $s$, and number of moles of each component, $\Gamma_{i}(i=1 \ldots \kappa)$. The location of the reference surface may now be specified by setting any one of these quantities equal to zero. Each of these possibilities then constitutes a convention. For a one-component system, it is convenient to choose the convention, $\Gamma=0$. In Figure 3 the application of this convention to a hypothetical variation of the mass density through the interfacial region is 
shown. For multicomponent systems, various conventions involving either one of the $\Gamma_{i}$, or a judicious combination of $\Gamma_{i}$, may be used ${ }^{12}$.

It is seen that the use of the reference surface concept permits the total quantity $M$ to be partitioned among three quantities. The sets of parameters represented by $M^{\alpha}$ and $M^{\beta}$, supplemented by the volumes $V^{\alpha}$ and $V^{\beta}$, respectively, constitute sets of homogeneous functions of order one. Similarly, the quantities $m \Omega$, supplemented by the area $\Omega$, form a set of homogeneous functions of order one. Such mathematical properties are essential in developing a rigorous thermodynamic analysis of the system which has been defined. At this stage of the analysis, however, the treatment is rigorous only if the area of $\mathscr{S}$ is taken to be very small.

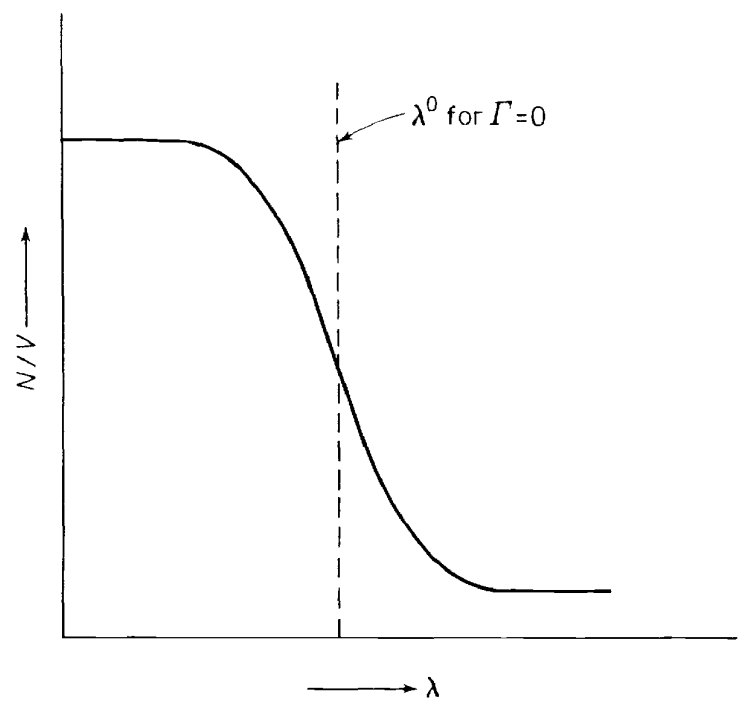

Figure 3. Schematic density profile for interfacial region

The motivation behind the procedures just described has often been interpreted in such a way that alternative formulations have been sought. In particular, the use of two dividing surfaces has been proposed. By this means a volume and a thickness of the interfacial region are defined. If the Gibbs approach is interpreted to imply the assignment of mass, energy and entropy to a fictitious surface, the use of two dividing surfaces would, of course, provide a more satisfactory approach. On the other hand, such an interpretation is not required. The dividing surface is not intended to represent the physical interface. It is instead merely a mathematical device and serves as a reference or fiducial surface. By means of such a surface the continuous variations of the densities of the extensive thermodynamic parameters can be compared with a discontinuous variation, yielding surface densities having required mathematical properties.

In fact, the apparent advantage of using two dividing surfaces is obviated by the corresponding need to introduce two conventions for locating the surfaces. One such convention would be to choose the second dividing surface 
to coincide with the first. The Gibbs formulation is then recovered. Also, it is not possible to assign a magnitude to the interfacial thickness by any means other than by adopting two conventions. Thus, this distance cannot have the physical significance which is intended.

\section{THE DIVIDING SURFACE AND THERMODYNAMIC WORK}

In order to interrelate the various thermodynamic quantities denoted by $M$ by a fundamental Gibbsian equation, it is necessary to develop a suitable formulation of the differential work which can be performed on the system $\mathscr{B}$. Clearly, this formulation will involve the displacement of the three surfaces, $\mathscr{S}^{\alpha}, \mathscr{S}^{\beta}$ and $\mathscr{S}_{N}$, which form the external boundaries of $\mathscr{B}$. In addition, the reference surface $\mathscr{S}$ is again found to play an essential role in the analysis.

Before proceeding to express the work received by the system in terms of geometric variables, however, it is useful to consider the nature of the thermodynamic potential directly related to such variables. Thus, according to the first law, for adiabatic changes in which the system $\mathscr{B}$ is closed, the differential work received by the system, $\mathrm{d} W$, is given by

$$
\mathrm{d} W=\mathrm{d} U ; \mathrm{d} S=\mathrm{d} N_{i}=0
$$

More generally, if $T$ denotes the temperature of the system and $\mu_{i}(i=1 \ldots \kappa)$ the set of chemical potentials associated with the components of the system,

$$
\mathrm{d} U=T \mathrm{~d} S+\sum_{i=1}^{\kappa} \mu_{i} \mathrm{~d} N_{i}+\mathrm{d} W
$$

Although $W$ is not itself a function of the thermodynamic state, there exists a thermodynamic potential, denoted as $\Phi$, which is obtained if equation 3.2 is simply integrated, using Euler's theorem on homogeneous first-order functions. Thus, integration of equation 3.2 yields

$$
\Phi=U-T S-\sum_{i=1}^{\kappa} \mu_{i} N_{i}
$$

The potential $\Phi$ is the free energy available for mechanical work at constant $T, \mu_{i}$. If $P$ denotes the pressure in a homogeneous fluid phase, the potential $\Phi$ for either of the homogeneous regions in the system $\mathscr{B}$ is given by

$$
\Phi^{j}=-P^{j} V^{j} \quad(j=\alpha, \beta)
$$

The corresponding expressions for the thermodynamic work done on such regions are:

$$
\mathrm{d} W^{j}=-P^{j} \mathrm{~d} V^{j} \quad(j=\alpha, \beta)
$$

The generalization of equations 3.4 to the entire system, as indicated above, involves the displacement of the surfaces forming the boundaries of $\mathscr{B}$. Appropriate geometrical transformations can then be introduced by a method $^{9}$ analogous to that used previously for spherical interfaces ${ }^{8,13}$. For an infinitesimal area, $\delta \Omega$, of the reference surface $\mathscr{S}$, this procedure yields the following expression for the work received,

$$
\mathrm{d}(\delta W)=-P^{\alpha} \mathrm{d}\left(\delta V^{\alpha}\right)-P^{\beta} \mathrm{d}\left(\delta V^{\beta}\right)+\gamma \mathrm{d}(\delta \Omega)+c(\delta \Omega) \mathrm{d} J
$$


Here, the intensive quantities $\gamma$ and $c$ are defined by the relationships :

$$
\begin{gathered}
\delta \Phi=-P^{\alpha} \delta V^{\alpha}-P^{\beta} \delta V^{\beta}+\gamma \delta \Omega \\
P^{\alpha}-P^{\beta}=\gamma J+c\left(2 K-J^{2}\right)
\end{gathered}
$$

It should be emphasized that the argument leading to equations 3.5 to 3.7 involves no more than two essential steps ${ }^{5,14}$. First, it is recognized that the variation $\mathrm{d}(\delta W)$ can be expressed solely in terms of the variations in a set of three independent geometrical variables. These variables are sufficient to specify the three types of volume change capable of doing work, i.e. changes resulting from the displacement of the boundary surfaces, $\mathscr{S}^{\alpha}, \mathscr{S}^{\beta}$ and $\mathscr{S}_{N}$. Secondly, suitable geometrical transformations are employed in order to express these variations in terms of the variables appearing in equation 3.5. In particular, at no stage is any principle of mechanics explicitly invoked.

On the other hand, the Gibbs reference surface $\mathscr{S}$ is directly involved in the geometrical transformations which are used. Thus, each of the five geometrical variables in equations 3.6 and 3.7 is defined only in terms of a particular reference surface. Hence, the magnitude of each quantity depends on the convention used to specify the location of $\mathscr{S}$. The form of equation 3.7 suggests that one such convention is given by the condition, $c=0$. The surface which corresponds to this convention is known as the 'surface tension'.

Since it is assumed that $\Phi$ is a function of state, i.e. $\Phi$ is determined for a given equilibrium state of the system, it follows from equation 3.6 that the interfacial tension, $\gamma$, is not a pure function of state but depends on the location of the reference surface $\mathscr{S}$. In fact, it can be shown that the parameter $c$ is related to this dependence by the following expression,

$$
c=\left(2 K-J^{2}\right)^{-1}\left(\partial \gamma / \partial \lambda^{0}\right) S, N_{i}, \mathscr{S}^{a}, \mathscr{S}^{a}, \mathscr{S}_{N}
$$

Thus, it follows from equation 3.7 that, unless $J=K=0$, the function $\gamma$ has a minimum when $\lambda^{0}$ corresponds to the surface of tension. For the case of a planar interfacial region, $\gamma$ is invariant with respect to the location of the reference surface $\mathscr{S}$.

Before proceeding further, two major difficulties may be noted. First, unless it is assumed at the outset that the reference surface is characterized by uniform curvatures, $J$ and $K$, over a finite area, $\Omega$, equations 3.5 to 3.7 provide no basis for regarding $\gamma$ and $c$ as uniform over such an area. That is, the analysis as developed to this point is restricted to interfacial regions for which the set of parallel reference surfaces is either planar, spherical or cylindrical in configuration. A second difficulty arises in connection with the location of the surface of tension. Within the context of the thermodynamic definition of work provided by equations 3.5 to 3.7 , the location of the surface of tension is only specified by the condition $c=0$. This convention, however, does not provide any information as to the proximity of this surface to the physical interface. In contrast, for a one-component system the convention $\Gamma=0$, as required by equation 2.1 , definitely locates the surface $\mathscr{S}$ within the nonhomogeneous interfacial region. 


\section{THE STRESS TENSOR AND CONDITION FOR HYDROSTATIC EQUILIBRIUM}

Both of the difficulties noted above arise because the Gibbsian thermodynamic approach is actually incomplete. The additional physical principle which is required in order to extend the traditional treatment is provided by an explicit consideration of the basic theorem of hydrostatics. The earlier literature devoted to the hydrostatic treatment of interfacial regions has been reviewed by Bakker ${ }^{15}$. However, the application of the condition for hydrostatic equilibrium to the critical problems noted, leading to a general phenomenological treatment of fluid-fluid interfacial regions, was first carried out by Buff $^{2,4,5,9}$.

The basic concept utilized in developing the hydrostatic analysis is that of the stress tensor. This quantity provides a convenient mathematical form for representing the forces or stress vectors acting on a small volume element of matter. In the case of a fluid phase at rest, the off-diagonal components vanish, and if the fluid is also isotropic, as in a bulk homogeneous fluid, the diagonal components are identical and, except for sign, are equal to the fluid pressure. In the nonhomogeneous interfacial region, on the other hand, the diagonal components of the stress tensor are not identical. Clearly, these components are best defined in terms of tangential and normal directions corresponding to a Gibbs reference surface $\mathscr{S}$. The fluid character of the interfacial region ensures isotropy in a two-dimensional sense, and consequently the two tangential components will be equal. Thus, with respect to any reference surface $\mathscr{S}$, the stress tensor, $\sigma$, can be written as

$$
\boldsymbol{\sigma}=\sigma_{T}\left(\mathbf{e}_{1} \mathbf{e}_{1}+\mathbf{e}_{2} \mathbf{e}_{2}\right)+\sigma_{N} \mathrm{nn}
$$

Here, $\sigma_{T}$ and $\sigma_{N}$ represent the tangential and normal components, respectively, $\mathbf{e}_{1}$ and $\mathbf{e}_{2}$ are orthogonal unit tangent vectors, and $\mathbf{n}$ is the unit normal vector.

The condition for mechanical or hydrostatic equilibrium can now be easily expressed. This condition follows from the equation of motion of a fluid which is at rest and not subject to body forces, and therefore represents the principle of momentum balance for such a fluid. It is of interest to note that the explicit use of this condition is not ordinarily required in thermodynamics. For the system under consideration, the condition requires that the divergence of the stress tensor vanish,

$$
\nabla \cdot \sigma=0
$$

If now the continuous variations of $\sigma_{T}$ and $\sigma_{N}$ through the interfacial region are defined in terms of the $\lambda$ coordinate and associated with the corresponding variation of the mean curvature $J$ of the reference surface, equations 4.1 and 4.2 yield a differential equation. The integration of this equation can be carried out without reference to a discontinuous variation in the components $\sigma_{T}$ and $\sigma_{N}$. However, in order to arrive at a relationship corresponding to that obtained from the thermodynamic analysis, i.e. equation 3.7, such a variation must be introduced. Using the unit step function given by equation $2.2 b$, this variation can be defined as

$$
\sigma^{\alpha \beta}\left(\lambda^{0}\right)=-\{1-A(\lambda)\} P^{\alpha}-A(\lambda) P^{\beta}
$$


Also required are definitions of the quantities $\gamma$ and $c$ in terms of integrals similar in form to those given by equation 2.1 but involving the variation of the tangential component of the stress tensor. These definitions are given by $^{2,4,9}$ :

$$
\begin{gathered}
\gamma=\int_{\lambda^{\alpha}}^{\lambda^{\beta}}\left\{\sigma_{T}(\lambda)-\sigma^{\alpha \beta}\left(\lambda^{0}\right)\right\} B\left(\lambda, \lambda^{0}\right) \mathrm{d} \lambda \\
c=\int_{\lambda^{\alpha}}^{\lambda^{\alpha}}\left\{\sigma_{T}(\lambda)-\sigma^{\alpha \beta}\left(\lambda^{0}\right)\right\}\left(\lambda-\lambda^{0}\right) L\left(\lambda, \lambda^{0}\right) \mathrm{d} \lambda
\end{gathered}
$$

where

$$
L\left(\lambda, \lambda^{0}\right)=1+J K\left(J^{2}-2 K\right)^{-1}\left(\lambda-\lambda^{0}\right)
$$

Thus, using equations 4.4 and 4.5 , it is found that the integration of equation 4.2 yields a form which is identical with equation 3.7. Furthermore, when equation 4.4 is differentiated with respect to $\lambda^{0}$ and equations 4.5 introduced, equation 3.8 is recovered. We may conclude, therefore, that the relationship expressed by equation 3.7 constitutes the condition for mechanical or hydrostatic equilibrium which is applicable to the system $\mathscr{B}$.

These results have a number of important applications. The first is the linking of the condition for mechanical equilibrium to the parameters appearing in the expression for the differential of thermodynamic work, equation 3.5. Also, equations 4.4 and 4.5 provide the appropriate interpretation of $\gamma$ and $c$ as Gibbs surface excess properties, defined in terms of a Gibbs reference surface.

In this connection, it is seen from equation 4.4 that the interfacial tension, $\gamma$, is not restricted to its traditional role as a scalar thermodynamic variable, but has also a vectorial interpretation. Thus, $\gamma$ represents the direct macroscopic force which an interfacial region exerts on its surroundings ${ }^{16}$. The confusion which has existed in the past with respect to this interpretation of $\gamma$ has led to a number of incorrect views relating to macroscopic surface phenomena. This confusion may also be in part responsible for the widelyheld notion that the parameter $\gamma$ should be incorporated in a 'true' chemical potential term characterizing the components within the interfacial region.

A further application of equations 4.5 is concerned with establishing the location of the surface of tension with respect to the physical interface. Since this particular reference surface is defined by the convention $c=0$, equation $4.5 \mathrm{a}$ yields

$$
\lambda^{0}=\frac{\int_{\lambda^{\alpha}}^{\lambda^{\beta}}\left\{\sigma_{T}(\lambda)-\sigma^{\alpha \beta}\left(\lambda^{0}\right)\right\} L\left(\lambda, \lambda^{0}\right) \lambda \mathrm{d} \lambda}{\int_{\lambda^{\alpha}}^{\lambda^{\beta}}\left\{\sigma_{T}(\lambda)-\sigma^{\alpha \beta}\left(\lambda^{0}\right)\right\} L\left(\lambda, \lambda^{0}\right) \mathrm{d} \lambda}, \text { for } c=0
$$

This result shows that the surface of tension is indeed located within the interfacial region.

Another important consequence follows from extending to the function $\sigma_{T}(\lambda)$ the same assumption of two-dimensional homogeneity which was applied previously to the densities represented by $\hat{M}(\lambda)$. On the basis of equation 4.4, then, the interfacial tension $\gamma$ may be taken as approximately uniform over the entire area $\Omega$ of any reference surface, as in the case of the excess quantities, $m$, defined by equation 2.1 .

These conclusions may be used to arrive at some further important results. First, since the pressures $P^{\alpha}$ and $P^{\beta}$ are invariant with respect to the choice 


\section{J. C. MELROSE}

of a normal direction through the reference surface $\mathscr{S}$, it follows from equation 3.7 that the surface of tension is characterized by a uniform value of the product of $\gamma$ and the mean curvature $J$. Consequently, $J$ is very nearly uniform for this particular reference surface. As has been seen, the surface of tension lies within the extremely thin nonhomogeneous region, and therefore other reference surfaces lying within the physical interface will vary only slightly from this condition. This constitutes the restriction on the variation of $J$ over the area $\Omega$ of any reference surface, to which reference was made previously (Section 2). Also, the equilibrium configuration of the physical interface is now seen to be characterized by a condition of essentially uniform mean curvature.

A further consequence of the two-dimensional homogeneity assumed for the function $\sigma(\lambda)$ is that, to nearly the same degree of approximation, the parameter $c$ may be regarded as uniform over a finite area $\Omega$. To within this degree of approximation, then, it becomes possible to extend the validity of equations 3.5 to 3.7 to a finite area, $\Omega$, of any reference surface.

\section{FUNDAMENTAL EQUATIONS FOR EXCESS QUANTITIES}

The fundamental Gibbsian differential equation which is applicable to the system $\mathscr{B}$ may now be written. Extending equation 3.5 to finite area and combining this result with equation 3.2 , we obtain

$$
\mathrm{d} U=T \mathrm{~d} S+\sum_{i=1}^{\kappa} \mu_{i} \mathrm{~d} N_{i}-P^{\alpha} \mathrm{d} V^{\alpha}-P^{\beta} \mathrm{d} V^{\beta}+\gamma \mathrm{d} \Omega+c \Omega \mathrm{d} J
$$

Similarly, equation 3.6 may be combined with equation 3.3. Differentiating the resulting expression and applying equation 5.1 then gives a GibbsDuhem equation in the form.

$$
V^{\alpha} \mathrm{d} P^{\alpha}+V^{\beta} \mathrm{d} P^{\beta}=S \mathrm{~d} T+\sum_{i=1}^{\kappa} N_{i} \mathrm{~d} \mu_{i}+\Omega \mathrm{d} \gamma-c \Omega \mathrm{d} J
$$

These results, together with equation 3.7, the condition for hydrostatic equilibrium, constitute the thermodynamic formalism suitable for analysing a variety of physical effects involving the system $\mathscr{B}$. In many instances, however, the effect of temperature, pressure and chemical composition on the interfacial tension is of primary concern. It is then useful to develop a formalism more directly applicable to this situation. Following Defay et al. $^{3}$, the Gibbs excess properties, as defined by equation 2.1 and to which this formalism applies, may be regarded as characterizing a 'non-autonomous system'.

In developing this formalism, it is necessary to introduce explicitly the conditions for thermal and material equilibrium to which the various states of the system $\mathscr{B}$ are subject. Thus, the temperature and chemical potentials which were introduced in writing equation 3.2 also apply ${ }^{1,3}$ to each of the homogeneous fluid regions $\alpha$ and $\beta$,

$$
\begin{gathered}
T=T^{\alpha}=T^{\beta} \\
\mu_{i}=\mu_{i}^{\alpha}=\mu_{i}^{\beta} \quad(i=1 \ldots \kappa)
\end{gathered}
$$


We then write equations 3.2 and 3.3 for each of the homogeneous regions. Introducing equations 3.4, these expressions yield the Gibbs-Duhem forms

$$
V^{j} \mathrm{~d} P^{j}=S^{j} \mathrm{~d} T+\sum_{i=1}^{\kappa} N_{i}^{j} \mathrm{~d} \mu_{i} \quad(j=\alpha, \beta)
$$

Subtracting equations 5.4 from equation 5.2, taking into account the appropriate relationships given by equation 2.6 , and dividing by the area of the reference surface then gives

$$
\mathrm{d} \gamma=-s \mathrm{~d} T-\sum_{i=1}^{\kappa} \Gamma_{i} \mathrm{~d} \mu_{i}+c \mathrm{~d} J
$$

Similarly, subtracting the two forms of equation 3.3 applicable to $\alpha$ and $\beta$ from the expression obtained by combining equations 3.3 and 3.6 yields

$$
u=T s+\sum_{i=1}^{\kappa} \mu_{i} \Gamma_{i}+\gamma
$$

These results define the formalism which interrelates the various Gibbs excess properties. For a planar interface, the final term in equation 5.5 is eliminated. Since in this case the intensive state of the system is determined by $\kappa$ variables, equation 5.5 still includes on the RHS one more term than is required. This is, of course, remedied by introducing the convention by which the Gibbs reference surface is located. For example, if component 1 is taken to be the solvent and if the $\Gamma_{1}=0$ convention is then adopted, equation 5.5 yields

$$
\mathrm{d} \gamma=-s \mathrm{~d} T-\sum_{i=2}^{\kappa} \Gamma_{i} \mathrm{~d} \mu_{i}
$$

This is the Gibbs adsorption equation. Its derivation has been the subject of much discussion in the literature ${ }^{17-19}$.

\section{CURVATURE DEPENDENCE OF INTERFACIAL TENSION}

The degree of approximation which is involved in extending the treatment developed in Sections 2-4 to finite areas is clearly related to the dependence of the interfacial tension on the mean curvature $J$. However, before discussing the nature of this dependence, it will be instructive to consider briefly the assumptions which are inherent in the experimental procedures used in measuring $\gamma$.

Nearly all conventional methods of measuring interfacial tension are based on the condition for hydrostatic equilibrium, equation 3.7. Implicitly, it is assumed that the appropriate dividing surface is the surface of tension. Due to the effect of the gravitational field, the pressure difference, $P^{\alpha}-P^{\beta}$, varies with the vertical coordinate. As a result, the mean curvature $J$ also varies with this coordinate. However, the curvatures involved are very small, and hence it is assumed that $\gamma$ is independent of the magnitude of $J$.

In systems of this type the boundary curve $\mathscr{C}$ is determined by the configuration of the surface of a solid phase, e.g. a cylindrical capillary, within which the system is enclosed, and by the condition for hydrostatic equilibrium applicable to the three-phase confluent zone ${ }^{4,16}$. The solid surface must be 


\section{J. C. MELROSE}

sufficiently smooth, as well as homogeneous with respect to composition and state of stress. Then, if its configuration is symmetrical with respect to the vertical axis, the interfacial region will be such that any reference surface $\mathscr{S}$ will also be symmetrical in this sense, i.e. a surface of revolution. Under these circumstances, numerical methods can be employed to obtain solutions to equation 3.7. It then becomes possible to relate measurements of the interface configuration to the fluid densities and to the interfacial tension.

In contrast to the situation involved in measuring $\gamma$, there are many cases of physical interest in which the curvatures are sufficiently high that gravitational distortion can be neglected. If the curvatures are extremely high, the question as to whether $\gamma$ is curvature dependent becomes a matter of some importance. As indicated above, this dependence is also of interest in assessing the magnitude of the approximation which is involved in extending the thermodynamic treatment to finite areas.

Since for a system which includes a curved interfacial region there are $\kappa+1$ independent intensive variables, one of the terms in equation 5.5 may again be eliminated by introducing a convention. For a one-component system, the curvature dependence is then represented by either of two relationships which follow from this expression,

$$
\begin{aligned}
\Gamma & =0 \text { convention }: \rightarrow(\partial \gamma / \partial J)_{T}=c \\
c & =0 \text { convention }: \rightarrow(\partial \gamma / \partial J)_{T}=-\Gamma(\partial \mu / \partial J)_{T}
\end{aligned}
$$

The further analysis of the problem, based on equations 6.1 , is due primarily to Tolman $^{20}$, Koenig ${ }^{7}$ and Buff ${ }^{2}$. This analysis utilizes a thermodynamic parameter which characterizes the thickness of the interface and is defined as the distance between the two dividing surfaces in question,

$$
\Delta \lambda=\lambda_{\Gamma=0}^{0}-\lambda_{c=0}^{0}
$$

It is then found that for the case of spherical interfaces $\left(J=2 K^{\frac{1}{2}}\right)$, the derivatives in equations 6.1 can be expressed by means of a polynomial in the variable $\Delta \lambda$. Retaining only the first-order term, it can be shown that

$$
\left(\frac{\partial \log \gamma}{\partial \log J}\right)_{T, \Gamma=0}=\left(\frac{\partial \log \gamma}{\partial \log J}\right)_{T, c=0} \simeq-(\Delta \lambda) J_{c=0}
$$

As Buff ${ }^{2,4}$ has pointed out, the variable $\Delta \lambda$ is in principle also curvature dependent. Consequently, the integration of equation 6.3 yields only the first-order correction to the interfacial tension appropriate to a planar interface. Denoting the latter as $\gamma_{\infty}$ and the corresponding interfacial thickness parameter as $\Delta \lambda_{\infty}$, this integration gives

$$
\gamma \simeq \gamma_{\infty}\left(1-\Delta \lambda_{\infty} J\right)
$$

Since various lines of indirect evidence suggest that the thickness of an interfacial region corresponds to no more than five or six molecular diameters $^{3-5}$, a reasonable estimate of the absolute magnitude of $\Delta \lambda_{\infty}$ is 2 to $4 \AA$. If this estimate is accepted, equation 6.4 indicates that for spherical interfaces, the effect of curvature becomes appreciable only if the radius of curvature is less than about $500 \AA$.

The sign of $\Delta \lambda_{\infty}$ can also be predicted. For a one-component system the 
surface of tension lies on the higher density or liquid side of the $\Gamma=0$ surface $^{2,4}$. If phase $\alpha$ is taken to be the liquid phase, $\Delta \lambda_{\infty}$ will then be positive in sign. The surface tension of a very small liquid drop is then, according to equation 6.4, less than the tension of a planar interface. Conversely, if phase $\alpha$ is the vapour phase, $\Delta \lambda_{\infty}$ is negative. The surface tension for a small bubble of vapour is correspondingly enhanced.

The experimental techniques used in measuring $\gamma$ involve curvatures such that the correction term in equation 6.4 is of the order of 0.001 per cent or less. Thus, the assumptions ordinarily used in interpreting measurements of $\gamma$ introduce errors of negligible magnitude. On the other hand, for applications involving interfaces of high curvature, these corrections can become important ${ }^{9}$. It should be noted that the estimate provided by equation 6.4 is applicable only to interfaces which are spherical in form. That is, the first-order treatment of curvature dependence so far developed does not necessarily extend to interfaces of more complicated shape.

\section{CONCLUSIONS}

The thermodynamic treatment of fluid-fluid interfacial regions which has been outlined above utilizes several essential concepts. The first is the notion of a Gibbsian dividing or reference surface. In addition, the mathematical description of curved surfaces provided by differential geometry can be used to extend the Gibbsian treatment of excess properties to interfacial regions which are neither flat, cylindrical, nor spherical. The thermodynamic work associated with changes in interfacial configuration is precisely formulated only through the use of these techniques. If the condition for hydrostatic equilibrium is then explicitly taken into account, the required interpretation of the parameters defined by this formulation can be established. Thus, by introducing this principle, the logical structure of the thermodynamic treatment applicable to fluid interfacial systems is completed.

\section{ACKNOWLEDGEMENT}

Appreciation is expressed to Mobil Research and Development Corporation for permission to publish this paper.

\section{REFERENCES}

1 J. W. Gibbs, Scientific Papers, Vol. I, pp. 219-331. Longmans: London (1906); Dover reprint: New York (1961).

${ }^{2}$ F. P. Buff, J. Chem. Phys. 23, 419 (1955); 25, 146 (1956).

${ }^{3}$ R. Defay, I. Prigogine, A. Bellemans and D. H. Everett, Surface Tension and Adsorption. Wiley: New York (1966).

${ }^{4}$ F. P. Buff, The Theory of Capillarity in Handbuch der Physik, Vol. X, pp 281-304. Springer: Berlin (1960).

${ }^{5}$ S. Ono and S. Kondo, Molecular Theory of Surface Tension in Liquids in Handbuch der Physik, Vol. X, pp 134-280. Springer: Berlin (1960).

6 R. C. Tolman, J. Chem. Phys. 16, 758 (1948).

7 F. O. Koenig, J. Chem. Phys. 18, 449 (1950).

8 T. L. Hill, J. Chem. Phys. 19, 1203 (1951).

T. L. Hill, J. Phys. Chem. 56, 526 (1952).

9 J. C. Melrose, Ind. Eng. Chem. 60 (No. 3), 53 (1968). 


\section{J. C. MELROSE}

${ }^{10}$ L. P. Eisenhart, Treatise on the Differential Geometry of Curves and Surfaces. Ginn : New York (1909); Dover reprint: New York (1960).

11 E. Kreysig, Differential Geometry. University of Toronto Press: Toronto (1959).

12 E. A. Guggenheim and N. K. Adam, Proc. Roy. Soc. A, 139, 218 (1933).

${ }^{13}$ S. Kondo, J. Chem. Phys. 25, 662 (1956).

14 E. A. Guggenheim, Research, Lond. 10, 478 (1959).

$15 \mathrm{G}$. Bakker, Kapillarität und Oberflächenspannung in Handbuch der Experimentalphysik, Vol. VI. Akademische Verlagsgesellschaft: Leipzig (1928).

16 F. P. Buff and H. Saltsburg, J. Chem. Phys. 26, 23, 1526 (1957).

17 F. O. Koenig and R. C. Swain, J. Chem. Phys. 1, 723 (1933).

18 E. A. Guggenheim, J. Chem. Phys. 4, 689 (1936).

19 G. Scatchard, J. Phys. Chem. 66, 618 (1962).

20 R. C. Tolman, J. Chem. Phys. 17, 333 (1949). 